\title{
Characterization of twenty-six microsatellite markers for the tropical pioneer tree species Cecropia insignis Liebm (Urticaceae)
}

\author{
Na Wei $\cdot$ Christopher W. Dick
}

Received: 13 June 2014/ Accepted: 7 July 2014/Published online: 17 July 2014

(C) Springer Science+Business Media Dordrecht 2014

\begin{abstract}
Cecropia insignis is an ecologically important Neotropical pioneer tree and major vertebrate food source. Although this species is relatively common in faunally intact tropical rainforests, its population dynamics may be negatively impacted by hunting of seed-dispersing animals. To better understand gene flow and regeneration dynamics in $C$. insignis, we characterized 26 microsatellite markers in a population sampled from Barro Colorado Island, Panama. Eleven loci of $\geq 3$ alleles were tested on 48 individuals, whereas the remaining 15 loci of two alleles were tested on 12 individuals. Allelic richness ranged from 2 to 9 per locus. Observed and expected heterozygosity averaged 0.478 and 0.440 respectively. Polymorphism information content was between 0.141 and 0.757 . Only two loci exhibited deviation from Hardy-Weinberg proportions.
\end{abstract}

Keywords Cecropia insignis - Microsatellite markers . Tropical tree $\cdot$ Seed dispersal

Cecropia insignis is a dioecious, gap-dependent canopy tree species distributed broadly in lowland moist forests of Central and northern South America. It provides important food resources (e.g., leaves, nectar, fruits) for forestdwelling animals. Although Cecropia trees represent one of the few primarily wind-pollinated taxa, its seed dispersal

\footnotetext{
N. Wei $(\bowtie) \cdot$ C. W. Dick

Department of Ecology and Evolutionary Biology, University of Michigan, 830 North University Avenue, Ann Arbor,

MI 48109-1048, USA

e-mail:weina@umich.edu

C. W. Dick

Smithsonian Tropical Research Institute,

PO Box 0843-03092, Balboa, Ancón, Republic of Panama
}

is mediated by vertebrates including large birds and monkeys. Hunting pressures are increasingly threatening the persistence of such seed-dispersing vertebrates, and have measurably altered tropical forest dynamics (Terborgh et al. 2008). We developed twenty-six polymorphic microsatellite markers for $C$. insignis to evaluate the impact of hunting and other anthropogenic changes on gene flow and regeneration in this species.

Methods used to obtain genomic data using circular consensus sequencing of Pacific Biosciences (PacBio) are described by Wei et al. (in press). Briefly, a PacBio 500-bp SMRTbell library was established from the genomic DNA of one $C$. insignis tree, and then sequenced using four SMRT cells with C2 chemistry. In total, 198,989 circular consensus reads were generated. A quality-control step (for details, see Wei et al. in press) was performed before searching for microsatellite loci and designing primers in QDD v2.1 (Meglécz et al. 2010). In total, 512 microsatellites loci were retrieved. From the pure (non-interrupted) microsatellite loci $(n=404)$, we synthesized 69 primer pairs (38 di-, 30 tri-, and 1 tetra-nucleotide motifs).

For marker validation, we isolated genomic DNA from 48 reproductive-sized trees of $C$. insignis growing on Barro Colorado Island, Panama. We adjusted the use of DNeasy Plant Mini Kit (QIAGEN, Valencia, California, USA) for high-throughput DNA isolation by replacing DNA binding columns with E-Z ${ }^{\circledR} 96$ DNA Plates (Omega Bio-Tek, Norcross, Georgia, USA). After an initial screening of primer amplification on three individuals, polymorphic loci were tested on another 9 samples. Then microsatellite loci showing $\geq 3$ alleles based on these 12 samples were scored on an additional 36 individuals. PCRs were carried out as follows: $94{ }^{\circ} \mathrm{C}$ for $4 \mathrm{~min}$; 28 cycles of $94{ }^{\circ} \mathrm{C}$ for $30 \mathrm{~s}$, $59{ }^{\circ} \mathrm{C}$ (decreasing $0.2{ }^{\circ} \mathrm{C}$ per cycle) for $40 \mathrm{~s}$ and $72{ }^{\circ} \mathrm{C}$ for $60 \mathrm{~s} ; 10$ cycles of $94{ }^{\circ} \mathrm{C}$ for $30 \mathrm{~s}, 53{ }^{\circ} \mathrm{C}$ for $40 \mathrm{~s}$ and $72{ }^{\circ} \mathrm{C}$ 
Table 1 Characteristics of 26 microsatellite markers developed in $C$. insignis

\begin{tabular}{|c|c|c|c|c|c|c|c|c|c|}
\hline Locus & Primer sequence $\left(5^{\prime}-3^{\prime}\right)^{\mathrm{a}}$ & Motif & $\begin{array}{l}\text { Size } \\
\text { range }\end{array}$ & $A$ & $H_{\mathrm{O}}$ & $H_{\mathrm{E}}$ & PIC & $\begin{array}{l}\text { Sample } \\
\text { size }\end{array}$ & $\begin{array}{l}\text { Accession } \\
\text { no. }\end{array}$ \\
\hline CEC_08 & $\begin{array}{l}\text { F: CTGCAATTGACTTGCCACAC } \\
\text { R: GGTGTGAAATGAAAGTGACCC }\end{array}$ & $(\mathrm{AAG})_{11}$ & $149-206$ & 5 & 0.771 & 0.642 & 0.593 & 48 & KF680367 \\
\hline CEC_10 & $\begin{array}{l}\text { F: ATTGCTCGTGCAACCAAAG } \\
\text { R: TTGTGCCATGTTAATAGCCC }\end{array}$ & $(\mathrm{AAT})_{8}$ & $258-285$ & 5 & 0.596 & 0.565 & 0.523 & 48 & KF680369 \\
\hline CEC_12 & $\begin{array}{l}\text { F: TTCCAATCCGGAGATAAACG } \\
\text { R: AAGCAAGAATCTCAAAGCCG }\end{array}$ & $(\mathrm{AAG})_{10}$ & $110-128$ & 4 & 0.708 & 0.581 & 0.524 & 48 & KF680371 \\
\hline CEC_17 & $\begin{array}{l}\text { F: TTCTTGATCGTGTTTGCTGC } \\
\text { R: AAATGTTCAAGGCATTGGTTC }\end{array}$ & $(\mathrm{AAT})_{7}$ & $115-127$ & 4 & 0.458 & 0.425 & 0.364 & 48 & KF680376 \\
\hline CEC_37 & $\begin{array}{l}\text { F: CAAGAGATGCGTCGAGAGTG } \\
\text { R: GGCAATCAATTTGCGTAACC }\end{array}$ & $(\mathrm{AG})_{16}$ & $151-157$ & 4 & 0.479 & 0.545 & 0.466 & 48 & KF680388 \\
\hline CEC_43 & $\begin{array}{l}\text { F: TTCGTGTATGAGGACAACGAG } \\
\text { R: AATTCCACGAGGAAGCAGAG }\end{array}$ & $(\mathrm{AG})_{14}$ & $293-317$ & 5 & 0.583 & 0.688 & 0.624 & 48 & KF680393 \\
\hline CEC_45 & $\begin{array}{l}\text { F: TTTACCAAACCCAATTCCC } \\
\text { R: ATTCTCAGCAAGTTCCCAGC }\end{array}$ & $(\mathrm{AG})_{13}$ & $118-152$ & 9 & 0.875 & 0.785 & 0.757 & 48 & KF680394 \\
\hline CEC_46 & $\begin{array}{l}\text { F: AGTACAACACCCGGATCGAC } \\
\text { R: TCGAATATAACGCCTCTCGC }\end{array}$ & $(\mathrm{AG})_{13}$ & $112-136$ & 8 & 0.604 & 0.528 & 0.503 & 48 & KF680395 \\
\hline CEC_56 & $\begin{array}{l}\text { F: TGGCCTTCTTGAGTTGTTTG } \\
\text { R: TCAGCCACTCTCACTCTTCG }\end{array}$ & $(\mathrm{AC})_{10}$ & 193-201 & 3 & 0.625 & 0.539 & 0.447 & 48 & KF680402 \\
\hline CEC_61 & $\begin{array}{l}\text { F: TCCAAGTAACATCCTCTCCСTC } \\
\text { R: TCCCTCAGAAAGCGAAGAAC }\end{array}$ & $(\mathrm{AG})_{10}$ & $115-121$ & 3 & 0.188 & 0.205 & 0.188 & 48 & KF680406 \\
\hline CEC_64 & $\begin{array}{l}\text { F: TTTGTCTTTGGCTTTGGACC } \\
\text { R: CAACCTTTGCAAATTGGTCTAC }\end{array}$ & $(\mathrm{AC}) 9$ & $145-155$ & 4 & 0.542 & 0.536 & 0.497 & 48 & KF680408 \\
\hline CEC_15 & $\begin{array}{l}\text { F: ACCAGAGCCTTGAACAATCC } \\
\text { R: TTCTTTGGACGAGAAATCGG }\end{array}$ & $(\mathrm{AAG})_{7}$ & $119-122$ & 2 & 0.167 & 0.278 & 0.239 & 12 & KF680374 \\
\hline CEC_22 & $\begin{array}{l}\text { F: CCGCATGGATAATTTCTCTTC } \\
\text { R: ACATCGTTGCATGAGCTTTG }\end{array}$ & $(\mathrm{AAT})_{8}$ & 204-207 & 2 & 0.333 & 0.375 & 0.305 & 12 & KF680381 \\
\hline CEC_31 & $\begin{array}{l}\text { F: GGGTGTATGCTCTCACACTTG } \\
\text { R: TCCATGATATGGTTTGGGTG }\end{array}$ & $(\mathrm{AAT})_{7}$ & $129-138$ & 2 & 0.333 & 0.278 & 0.239 & 12 & KF680386 \\
\hline CEC_34 & $\begin{array}{l}\text { F: TTAGGACTACTGCCTTCGCAC } \\
\text { R: TATTGAGGCATGGAGGCTTG }\end{array}$ & $(\mathrm{AC})_{19}$ & $153-163$ & 2 & 0.417 & 0.330 & 0.276 & 12 & KF680387 \\
\hline CEC_38 & $\begin{array}{l}\text { F: TTACAGAGCATTGTGACCCG } \\
\text { R: TGATGGAAGCTCTGAAGCAC }\end{array}$ & $(\mathrm{AG})_{15}$ & $159-161$ & 2 & 0.500 & 0.486 & 0.368 & 12 & KF680389 \\
\hline CEC_40 & $\begin{array}{l}\text { F: TTATGGGCAACTACGGCTTC } \\
\text { R: CCATGTTCTAAACAATGTGTCC }\end{array}$ & $(\mathrm{AG})_{15}$ & $121-125$ & 2 & 0.500 & 0.375 & 0.305 & 12 & KF680390 \\
\hline CEC_41 & $\begin{array}{l}\text { F: TGAGCAAGCTGGAAAGGAAG } \\
\text { R: TGCAAACCCAGCTATAAATGC }\end{array}$ & $(\mathrm{AG})_{15}$ & $156-166$ & 2 & 0.583 & 0.413 & 0.328 & 12 & KF680391 \\
\hline CEC_49 & $\begin{array}{l}\text { F: GAATTGCACATTGCCCTCTC } \\
\text { R: CTCCGGTCTCTTCCTTCCC }\end{array}$ & $(\mathrm{AG})_{12}$ & 116-118 & 2 & 0.417 & 0.330 & 0.276 & 12 & KF680397 \\
\hline CEC_52 & $\begin{array}{l}\text { F: ACCTTTGACCGTGGGATTC } \\
\text { R: TGGTTGTCAAACTGTAAGGCAG }\end{array}$ & $(\mathrm{AC})_{10}$ & $126-132$ & 2 & $1.000 * * *$ & 0.500 & 0.375 & 12 & KF680398 \\
\hline CEC_53 & $\begin{array}{l}\text { F: GGCTGAGAGCTTTGGAGATG } \\
\text { R: ACACTGTAGCAGAGCGGAGC }\end{array}$ & $(\mathrm{AG})_{10}$ & $142-150$ & 2 & 0.250 & 0.330 & 0.276 & 12 & KF680399 \\
\hline CEC_59 & $\begin{array}{l}\text { F: CCTCGGTGACCTTGAACTTG } \\
\text { R: AAGAAACCCTTCAATCTCTGC }\end{array}$ & $(\mathrm{AG})_{10}$ & $154-156$ & 2 & 0.167 & 0.153 & 0.141 & 12 & KF680404 \\
\hline CEC_60 & $\begin{array}{l}\text { F: CTCAGCATAGATCTCGTTGCC } \\
\text { R: TCTACTCAACAACCCGACCC }\end{array}$ & $(\mathrm{AG})_{10}$ & $184-186$ & 2 & 0.250 & 0.413 & 0.328 & 12 & KF680405 \\
\hline
\end{tabular}


Table 1 continued

\begin{tabular}{|c|c|c|c|c|c|c|c|c|c|}
\hline Locus & Primer sequence $\left(5^{\prime}-3^{\prime}\right)^{\mathrm{a}}$ & Motif & $\begin{array}{l}\text { Size } \\
\text { range }\end{array}$ & $A$ & $H_{\mathrm{O}}$ & $H_{\mathrm{E}}$ & $\mathrm{PIC}$ & $\begin{array}{l}\text { Sample } \\
\text { size }\end{array}$ & $\begin{array}{l}\text { Accession } \\
\text { no. }\end{array}$ \\
\hline CEC_62 & $\begin{array}{l}\text { F: GTTTGGTGGGTTCACATGG } \\
\text { R: CGATGTGTCACACTTGGGTC }\end{array}$ & $(\mathrm{AG})_{10}$ & $115-117$ & 2 & 0.583 & 0.413 & 0.328 & 12 & KF680407 \\
\hline CEC_65 & $\begin{array}{l}\text { F: TGAGGAATCTCCAAGGGAAG } \\
\text { R: TCAGTGATTGGACTTCTGTTCC }\end{array}$ & $(\mathrm{AC})_{9}$ & $117-121$ & 2 & $0.167 *$ & 0.444 & 0.346 & 12 & KF680409 \\
\hline CEC_67 & $\begin{array}{l}\text { F: CTTGAAACCGGCTCCTGAAC } \\
\text { R: TCGGGAATGGAAATAAATATGC }\end{array}$ & $(\mathrm{AG})_{9}$ & $157-163$ & 2 & 0.333 & 0.278 & 0.239 & 12 & KF680411 \\
\hline
\end{tabular}

$A=$ number of alleles per locus; $H_{\mathrm{O}}=$ observed heterozygosity; $H_{\mathrm{E}}=$ expected heterozygosity; PIC $=$ polymorphism information content

Significant deviation from Hardy-Weinberg proportions at $P<0.05(*)$ and $P<0.001$ (***)

${ }^{a}$ M13 tail (TGTAAAACGACGGCCAGT) attached to the $5^{\prime}$ end of individual forward primers

for $60 \mathrm{~s}$; and $72{ }^{\circ} \mathrm{C}$ for $10 \mathrm{~min}$. Each 8 - $\mu \mathrm{L}$ PCR contained $1 \mu \mathrm{L}$ of $4 \mathrm{ng} / \mu \mathrm{L}$ DNA, $0.05 \mu \mathrm{L}$ of $1 \mu \mathrm{M}$ HEX-labeled or $1.5 \mu \mathrm{M} \quad$ FAM-labeled $\quad \mathrm{M} 13 \quad$ primer (TGTAAAACGACGGCCAGT), $0.12 \mu \mathrm{L}$ of $5 \mu \mathrm{M}$ M13tagged forward primer, $0.48 \mu \mathrm{L}$ of $5 \mu \mathrm{M}$ reverse primer, $0.8 \mu \mathrm{L}$ of $25 \mathrm{mM} \mathrm{MgCl}_{2}, 4 \mu \mathrm{L}$ of GoTaq Colorless Master Mix (Promega, Madison, Wisconsin, USA), and $1.55 \mu \mathrm{L}$ $\mathrm{H}_{2} \mathrm{O}$. PCR products of two loci labeled by different dyes were sized in a single lane on an ABI 3730 DNA Analyzer (Life Technologies, Carlsbad, California, USA). Alleles were then scored using GeneMarker v2.4.1 (SoftGenetics, State College, Pennsylvania, USA). Allelic richness, observed and expected heterozygosity, and Hardy-Weinberg equilibrium (HWE) were estimated using GenAlEx v6.5 (Peakall and Smouse 2012). Polymorphism information content (PIC) was assessed in PowerMarker v3.25 (Liu and Muse 2005).

We described here only the 26 polymorphic microsatellite loci. For the eleven markers screened on 48 individuals (Table 1), allelic richness averaged 5 per locus (range 3-9). $H_{\mathrm{O}}$ ranged from 0.188 to $0.875 ; H_{\mathrm{E}}$ varied between 0.205 and 0.785 . PIC was between 0.188 and 0.757 (mean $=0.499)$. All of these eleven loci conformed to HWE. For the fifteen markers showing two alleles and tested on 12 individuals (Table 1), observed and expected heterozygosity averaged 0.400 and 0.360 respectively. PIC was between 0.141 and 0.375 . Two of the 15 loci (CEC_52 and CEC_65) deviated from Hardy-Weinberg expectations.

Acknowledgments This work was supported by a CTFS-ForestGEO grant from Smithsonian Tropical Research Institute and Center for Tropical Forest Science.

\section{References}

Liu KJ, Muse SV (2005) PowerMarker: an integrated analysis environment for genetic marker analysis. Bioinformatics 21(9):2128-2129. doi:10.1093/bioinformatics/bti282

Meglécz E, Costedoat C, Dubut V, Gilles A, Malausa T, Pech N, Martin JF (2010) QDD: a user-friendly program to select microsatellite markers and design primers from large sequencing projects. Bioinformatics 26(3):403-404. doi:10.1093/bioinfor matics/btp670

Peakall R, Smouse PE (2012) GenAlEx 6.5: genetic analysis in Excel. Population genetic software for teaching and research-an update. Bioinformatics 28(19):2537-2539. doi:10.1093/bioinformatics/ bts 460

Terborgh J, Nun ez-Iturri G, Pitman NCA, Valverde FHC, Alvarez P, Swamy V, Pringle EG, Paine CET (2008) Tree recruitment in an empty forest. Ecology 89(6):1757-1768

Wei N, Bemmels JB, Dick CW (in press) The effects of read length, quality and quantity on microsatellite discovery and primer development: from Illumina to PacBio. Mol Ecol Resour. doi:10. 1111/1755-0998.12245 